\title{
The Relationship Between Serum Lead Level and Neurobehavioral Performance
}

\author{
Omid Aminian ${ }^{1}$, Abbass Ali Saneian ${ }^{1}$ and Nazanin Izadi ${ }^{1}{ }^{, *}$ \\ ${ }^{1}$ Center for Research for Occupational Diseases, Tehran University of Medical Sciences, Tehran, Iran \\ "Corresponding author: Center for Research for Occupational Diseases, Tehran university of Medical Sciences, Tehran, Iran. Email: nazanin.izadi@gmail.com
}

Received 2018 March 09; Revised 2019 February 02; Accepted 2019 April 23.

\begin{abstract}
Background: Lead is one of the metals, which has an important role in the industry.

Objectives: We designed a study to assess the serum lead level in battery manufacturing workers and its association with neurobehavioral performance.

Methods: In this cross-sectional, 154 individuals who worked in a battery factory participated. Venous blood samples were collected for measuring blood lead levels by atomic absorption method. Three selected tests of neuropsychological performance approved by World Health Organization were conducted, including simple reaction time(SRT), Benton Visual Retention Test (BVRT), and Santa Ana manual dexterity test.

Results: The present study demonstrates a positive correlation between blood lead levels and work experience. The higher blood lead levels and longer work experience were associated with lower scores on the Benton Visual Test. Simple reaction time showed a significant relationship with the work experience.

Conclusions: The results of this study suggest that occupational exposure to lead can induce a neurobehavioral dysfunction in battery manufacturing workers. Thus neurobehavioral assessment in health surveillance of exposed workers can be effective in the early detection of cognitive impairment.
\end{abstract}

Keywords: Occupational Exposure, Lead, Battery Manufacturing, Neurobehavioral Performance

\section{Background}

Lead is one of the metals, which plays an important role in the industry. It is used in paint, ceramics, and also car battery manufacturing. The effect of lead on human health has always been one of the concerns of medical circles and one of the most important systems affected by lead is the central nervous system (CNS).

Neurotoxicity is a series of changes in the nervous system due to exposure to toxic agents, which may cause cognitive changes, memory impairments, and behavioral changes $(1,2)$. Contact with various chemical agents such as solvents and metals (such as lead and aluminum) can cause Psychomotor changes (3). The molecular mechanism of lead toxicity is mediated through the blockade of a receptor, $\mathrm{N}$-methyl-di-aspartate and the inactivation of Protein-G, which disrupts the functional relationship between astrocytes and endothelial cells (4).

Some studies have shown that exposure to lead causes changes in motor behaviors (5) and increased dopamine in basal ganglion (6) and reduces the activity of neurotransmitters and ultimately, affects cognitive functions (7).
There has also been a disturbance of simple reaction time (SRT), digit symbol (DS), memory impairment (8), motor deficiency (9), and low performance in the speech audiometry (SA). Other effects of lead on the nervous system are reduced function in the psycho-motor activities, performance, and learning. Some studies have defined the lead as a contributing factor in visuospatial information process deficiency and the lack of precise work (10). Moreover, the effect of lead on the prefrontal cortex has been described for cognitive impairment. Lead causes various degrees of damage to the nervous system, ranging from mild cognitive impairment to the risk of advanced dementia (11). Moreover, lead may indirectly increase blood pressure and thus dementia (12). Some studies have shown a relationship between blood lead concentrations and delayed motorway median nerve and it is known that the measurement of blood lead is useful as a tool for the early diagnosis of peripheral nerve disorders in the exposed workers (13).

It is difficult to judge the effects of lead on the central nervous system through clinical symptoms, and special para-clinical procedures, such as Functional MRI, are 
also not possible in most cases. Therefore, one of the proposed methods is the use of clinical neurobiological tests approved by the World Health Organization (NCTB-WHO) and some of these tests can identify the early stages of CNS damage by neurotoxic agents.

Most adult exposures are occupational and occur in lead-related industries, particularly in the battery manufacturing factories. There is little information about objective and accurate neurobehavioral tests due to heavy metals, especially lead exposure in adults. On the other hand, the results of neurobehavioral tests are different among various populations and there are limited studies about this topic in Iran.

\section{Objectives}

The purpose of this study was to use these tests on exposed workers to early detection of neurological disorders.

\section{Methods}

This cross-sectional study was conducted on battery factory workers in the west of Tehran province, the capital of Iran in 2016. One hundred and fifty-four battery factory workers were enrolled in this study. The inclusion criteria of the study were working in the production and maintenance unit and having at least 1 year of work experience. Subjects who had a previous history of head trauma, heart disease, diabetes, seizure, arrhythmias, used CNS drugs, having substance dependence and inadequate sleep in the previous night were excluded from the study. All workers were asked to give their permission by completing the consent form. Ethical approval for this study was obtained from the Ethics Committee of Tehran Medical School.

Demographic and occupational characteristics (type of occupation, duration of employment, educational level, and previous occupations) were recorded on the questionnaire. Medical examination and neurobehavioral performance tests were carried out by an occupational medicine specialist. The three selected tests of neuropsychological performance approved by the World Health Organization were conducted to evaluate neurological performance, which were as follows.

1) Simple reaction time (SRT): The measurement of response time is a classic method in neurotoxicology. The SRT shows how fast a person reacts and it requires sustained attention from the subject. In this 6-minute test, 64 red light stimuli appear at randomly varied intervals (110 second) on the computer screen and the subject should give fast responses by pressing one key; finally, we have the following scores: number of responses, number of omitted responses, mean reaction time, and standard deviation of the reaction time.
2) The Perdue pegboard is a test of manual dexterity, which requires rapid eye-motor coordination. The pegboard consists of a board with two parallel whole rows (right-hand and left-hand). The test involves a total of two trials, which requires the patient to place the pegs in the holes as quickly as possible in 30 seconds for each hand, and the final score is the number of pegs placed.

3) Benton Visual Retention test (BVRT) is a test of shortterm visual memory by measuring the ability to recognize geometrical patterns and memorize them. It consists of 20 cards presented as 10 pairs of two. After presenting the first card, the subject must recognize the identical pattern among the four shapes in the next card. The score is the number of correct patterns recognized $(0-10)$.

Venous blood samples were collected to measure blood serum lead levels. In fact, $2 \mathrm{cc}$ of the venous blood of these workers was collected at the beginning of the shift and before the start of work and gently mixed with EDTA anticoagulant powder, which is also lead chelating, then was transferred to the reference laboratory in a cool box. After centrifugation, blood serum levels were measured by atomic absorption and reported based on micrograms per deciliter.

These tests were carefully performed by a trained researcher and the results were recorded. Data were analyzed with SPSS version 18 software. Independent sample $t$-test was used for determination of association between quantitative variables. The Pearson correlation coefficient was used to examine the relationship between blood lead levels and working experience with neurobehavioral performance tests. P value $<0.05$ has been considered a significant association.

\section{Results}

In this study, 154 male workers from a battery factory participated in this study. The mean \pm SD age and work experience of subjects were $40.29 \pm 6.44$ and $15.48 \pm 5.03$ years, respectively. Shorter work experience, higher educational level, office workers, and morning workers had a significantly lower blood lead levels as shown in Table 1. Nevertheless, there was no relationship between age and blood lead levels.

The Pearson correlation coefficient was used to compare blood lead levels and work experience as a quantitative variable with neurobehavioral performance tests. Higher blood lead levels and longer work experience were associated with lower scores on the Benton visual test. Simple reaction time showed a significant relationship with the work experience (Table 2). Table 3 shows that Right Purdue pegboard and Benton Visual Tests had a significant relationship with the duration of employment in the factory. 


\begin{tabular}{|c|c|c|c|}
\hline Variables & No. (\%) & BLL mean $\pm S D$ & PValue \\
\hline Age, $y$ & & & 0.42 \\
\hline$\leq 40$ & $53(34.4)$ & $43.25 \pm 19.23$ & \\
\hline$>40$ & $51(65.6)$ & $45.71 \pm 17.68$ & \\
\hline Working history, y & & & 0.17 \\
\hline$\leq 15$ & $60(39)$ & $42.35 \pm 19.01$ & \\
\hline$>15$ & $94(61)$ & $46.47 \pm 17.59$ & \\
\hline Educational degree & & & 0.000 \\
\hline Below diploma & $63(40.9)$ & $53.78 \pm 14.59$ & \\
\hline$\geq$ diploma & $91(59.1)$ & $38.69 \pm 17.97$ & \\
\hline Job category & & & 0.000 \\
\hline Assembly line worker & $95(61.7)$ & $54.11 \pm 12.77$ & \\
\hline Office worker & $59(38.3)$ & $29.98 \pm 15.65$ & \\
\hline Working station & & & 0.000 \\
\hline Maintenance unit & $58(37.7)$ & $52.48 \pm 15.27$ & \\
\hline Assembling unit & $55(35.7)$ & $50.33 \pm 13$ & \\
\hline Others & $41(26.6)$ & $26.76 \pm 15.7$ & \\
\hline Exposure type & & & 0.003 \\
\hline Dust & $115(74.7)$ & $42.59 \pm 18.70$ & \\
\hline Mixed & $39(25.3)$ & $51.56 \pm 14.96$ & \\
\hline Shift work & & & 0.02 \\
\hline Morning & $119(77.8)$ & $43.00 \pm 18.50$ & \\
\hline Evening & $34(22.2)$ & $51.03 \pm 16.04$ & \\
\hline
\end{tabular}

Abbreviation: BLL, blood lead level.

${ }^{a}$ Independent sample $t$-test was used for comparison of BLL in all of the above variables, except for working station that the analysis was done with one-way ANOVA.

$\overline{\text { Table 2. Pearson Correlation Between Working History and Blood Lead Level With }}$ Neurobehavioral Performance Tests

\begin{tabular}{ccccc}
\hline $\begin{array}{l}\text { Neurobehavioral } \\
\text { tests }\end{array}$ & SRT & Pegboard Right & Pegboard left & BVRT \\
\hline Working history & & & & \\
\hline R & 0.10 & -0.22 & -0.11 & -0.34 \\
\hline P & 0.18 & 0.004 & 0.15 & 0.000 \\
\hline Blood lead level & & & & \\
\hline R & 0.13 & -0.028 & 0.05 & -0.18 \\
\hline$P$ & 0.09 & 0.72 & 0.54 & 0.023 \\
\hline
\end{tabular}

Abbreviations: BVRT, Benton Visual Retention Test; P, P value; r, coefficient correlation; SRT, simple reaction time.

\section{Discussion}

The present study evaluated the relationship between blood lead level in battery factory workers and its associ- ation with neuro-behavioral performance. In this study, no significant relationship was observed between age and blood lead and this could be attributed to the younger age of the studied subjects. However, in the study of Ravibabu et al., a significant relationship was found between age and serum levels (14). The present investigations showed a positive correlation between blood lead levels and years of employment and also a lower score of the Benton Visual Test. This implies that higher exposure to lead in terms of working years can slow down and delay the short-term visual memory.

The study of Chen et al. revealed that there was an important gap in neuronal function in workers with lead levels between 40 and $80 \mu \mathrm{g} / \mathrm{dL}$ and it slowed down the psychomotor performances, the processing failure of spatial visibility information, the responses of task implementation, and the lack of control attention (10). Schwartz et al. (15) demonstrated some evidence for longitudinal changes in blood lead levels with neurobehavioral decline. According to their study, exposed workers had more annual declines in verbal memory and visuoconstruction domains (15).

The results of a cross-sectional study conducted by Shih et al. (16) showed that low current blood lead levels were not associated with lower scores in cognitive function. However, the cumulative exposure was significantly associated with worse performance in all seven cognitive domains (16). The study by Barth et al. found that visuospatial abilities differed significantly between the exposed groups (17). The results of Abdolmaleki study showed that in workers in contact with lead, the prevalence of physical symptoms (31.9\%), anxiety (29.5\%), and social dysfunction (21.4\%) and depression (16.2\%), respectively (18).

Fenga et al. (19) stated that even in acceptable amounts, lead can cause some cognitive-behavioral problems such as tension, depression, invasion, fatigue, and turbulence. Their results were consistent with the latest scientific achievements, indicating that increased blood lead can be associated with measured cognitive abnormalities and also suggested that there was no safe blood level for the adverse effects of lead on neural function (19). In the investigation of 146 workers exposed to lead in the battery manufacturing industry, symbol digit test for the assessment of attention and perception was the only domain that had been affected by increased blood lead levels (BLL) (14). The study of Chand Basha demonstrated that exposure to lead can cause age-related behavioral changes (5). The results of the current study were consistent with a previous study, which showed no significant relationship between SRT and lead (20).

Despite our study, Krieg et al. reported that serum levels below $25 \mu \mathrm{g}$ had no effect on neurologic behavioral function (21). Weisskopf et al. found that patella lead levels 


\begin{tabular}{|c|c|c|c|c|c|c|}
\hline \multirow{2}{*}{ Neurobehavioral Tests } & \multicolumn{3}{|c|}{ Working History, y } & \multicolumn{3}{|c|}{ Blood Lead Level } \\
\hline & $<\mathbf{1 5}$ & $\geq 15$ & PValue & $<30$ & $\geq 30$ & P Value \\
\hline Simple reaction tests & $325.34 \pm 40.69$ & $336 \pm 56.96$ & 0.21 & $327.7 \pm 42.76$ & $333.21 \pm 53.98$ & 0.56 \\
\hline $\begin{array}{l}\text { Perdue pegboard } \\
\text { right }\end{array}$ & $15.43 \pm 1.60$ & $14.59 \pm 1.25$ & 0.001 & $14.87 \pm 1.11$ & $14.93 \pm 1.55$ & 0.81 \\
\hline Perdue pegboard left & $14.17 \pm 1.44$ & $13.89 \pm 1.39$ & 0.24 & $13.79 \pm 1.11$ & $14.07 \pm 1.49$ & 0.29 \\
\hline Benton Visual Test & $8.13 \pm 1.22$ & $7.27 \pm 1.44$ & 0.000 & $8.00 \pm 1.78$ & $7.47 \pm 1.26$ & 0.04 \\
\hline
\end{tabular}

${ }^{\mathrm{a}}$ Values are expressed as mean $\pm \mathrm{SD}$.

were significantly associated with a decline in Mini-Mental State Examination score over time, but there was no association with blood lead levels (22). A review of studies, which was done about lead exposure and cognitive function in adults, indicated that the impact of cumulative dose in former workers with past occupational lead exposure was stronger than blood lead levels; however, in the currently exposed workers, the association was stronger with blood lead levels. They suggest that the acute effects of recent lead exposure may attenuate the chronic effects of cumulative dose (23). One of the strengths of this study was the large sample size, which was less in other studies. This sample size helps to increase the external validity and generalize the results to the workers' community exposed to lead in the battery factory. Also, the use of objective and nonsubjective tests, and the measurement of high accuracy blood lead levels in the reference laboratory are among the other strengths of this study.

The limitation of this study was the implementation of the workers of one battery factory. Also, blood lead levels may also be due to exposure to some environmental exposure such as air pollution and there was no possibility of differentiation in our study. Lack of assessment of cumulative dose and body burden of lead by X-ray fluorescence method was another limitation. The causal relationship of our results is limited according to the type of this study. Therefore, we recommend implementing a prospective study to control the confounding effect.

\subsection{Conclusions}

In this study, according to regression analysis, there was a significant positive correlation between blood lead levels with working history, and also a borderline association with Benton Visual Tests (Table 4). This study suggests that occupational exposure to lead can induce a neurobehavioral dysfunction in the currently exposed workers in the battery manufacturing industry. Further investigations are needed to define whether cumulative lead exposure can affect neurobehavioral performance. Therefore, implementing the neurobehavioral assessment in the preemployment and periodic health surveillance of exposed workers may be effective in the early detection of cognitive impairment.

\begin{tabular}{|c|c|c|c|}
\hline Variables & B & Sig. & Odds ratio CI (95\%) \\
\hline Age & 0.047 & 0.377 & $1.05(0.94-1.16)$ \\
\hline Working history & 0.010 & 0.043 & $1.01(1.001-1.019)$ \\
\hline Benton & -0.826 & 0.058 & $0.44(0.17-1)$ \\
\hline Peg board right & 0.199 & 0.685 & $1.22(0.46-3.2)$ \\
\hline Shift work & 0.998 & 0.114 & $2.71(0.79-9.36)$ \\
\hline Smoking & 1.972 & 0.062 & $7.18(0.9-57)$ \\
\hline Constant & -63.990 & 0.377 & 0.000 \\
\hline
\end{tabular}

\section{Acknowledgments}

We want to appreciate the cooperation of workers in this study.

\section{Footnotes}

Authors' Contribution: Study concept and design: Omid Aminian, Nazanin Izadi, and Abbass Ali Saneian. Acquisition of data: Saneian. Analysis and interpretation of data: Nazanin Izadi. Drafting of the manuscript: Abbass Ali Saneian. Critical revision of the manuscript for important intellectual content: Omid Aminian, Nazanin Izadi. Statistical analysis: Nazanin Izadi. Administrative, technical, and material support: Omid Aminian, Abbass Ali Saneian. Study supervision: Omid Aminian, Nazanin Izadi.

Conflict of Interests: There is no conflict of interest with any stakeholders.

Ethical Approval: Ethical approval for this study was obtained from the Ethics Committee of Tehran Medical School.

Funding/Support: This study was conducted with the support of Tehran University of Medical Sciences. 
Informed Consent: All workers were asked to give their permission by completing the consent form.

\section{References}

1. Han DY, Hoelzle JB, Dennis BC, Hoffmann M. A brief review of cognitive assessment in neurotoxicology. Neurol Clin.2011;29(3):581-90. doi: 10.1016/j.ncl.2011.05.008. [PubMed: 21803211].

2. Mason LH, Mathews MJ, Han DY. Neuropsychiatric symptom assessments in toxic exposure. Psychiatr Clin North Am. 2013;36(2):201-8. doi: 10.1016/j.psc.2013.02.001. [PubMed: 23688687].

3. Aminian O, Hashemi S, Sadeghniiat-Haghighi K, Shariatzadeh A, Naseri Esfahani AH. Psychomotor effects of mixed organic solvents on rubber workers. Int J Occup Environ Med. 2014;5(2):78-83. [PubMed: 24747998].

4. Brochin RLS, Phillips D, Shepard N, Zisa D, Angerio AGUJHS. The cellular effect of lead poisoning and its clinical picture. Georgetoen Univ J Health Sci. 2008;5(2):79-85.

5. Chand Basha D, Saya Reddy N, Usha Rani M, Rajarami Reddy G. Age related changes in aminergic system and behavior following lead exposure: protection with essential metal supplements. Neurosci Res. 2014;78:81-9. doi: 10.1016/j.neures.2013.09.007. [PubMed: 24064391].

6. Szczerbak G, Nowak P, Kostrzewa RM, Brus R. Maternal lead exposure produces long-term enhancement of dopaminergic reactivity in rat offspring. Neurochem Res. 2007;32(10):1791-8. doi: 10.1007/s11064-0079306-0. [PubMed: 17404837].

7. Leret ML, Garcia-Uceda F, Antonio MT. Effects of maternal lead administration on monoaminergic, GABAergic and glutamatergic systems. Brain Res Bull. 2002;58(5):469-73. doi: 10.1016/s0361-9230(02)00819-5. [PubMed: 12242099].

8. Carta P, Carta R, Aru G, Polizzi M, Gaviano L, Salis S, et al. [Neurobehavioural testing in lead smelter workers]. G Ital Med Lav Ergon. 2005;27(3):285-9. Italian. [PubMed:16240575].

9. Muzi G, Murgia N, Dell'Omo M, Fiordi T, Sposini F, Argentino A, et al. [Effects of inorganic lead exposure on the autonomic nervous system and on the variability of heart rate among workers at a battery plant]. G Ital Med Lav Ergon. 2005;27 Suppl 1:46-50. Italian. [PubMed: 15915655].

10. Chen SS, Chen TJ, Lin CH, Tseng YT, Lai SL. Neurobehavioral changes in Taiwanese lead-exposed workers.JOccup Environ Med.2005;47(9):9028. doi: 10.1097/01.jom.0000169511.23124.6e. [PubMed:16155475].

11. Chertkow H, Massoud F, Nasreddine Z, Belleville S, Joanette Y, Bocti C, et al. Diagnosis and treatment of dementia: 3. Mild cognitive impairment and cognitive impairment without dementia. CMAJ. 2008;178(10):1273-85. doi: 10.1503/cmaj.070797. [PubMed: 18458258]. [PubMed Central: PMC2335177].

12. Rapisarda V, Ledda C, Ferrante M, Fiore M, Cocuzza S, Bracci M, et al. Blood pressure and occupational exposure to noise and lead $(\mathrm{Pb})$
A cross-sectional study. Toxicol Ind Health. 2016;32(10):1729-36. doi: 10.1177/0748233715576616. [PubMed: 25883097].

13. Reihani Kermani H, Niktab AR. The relationship between blood lead concentration and electroneurographic findings in lead-exposed subjects. J Qazvin Univ Med Sci. 2005;8(4):27-31.

14. Ravibabu K, Barman T, Rajmohan HR. Serum neuron-specific enolase, biogenic amino-acids and neurobehavioral function in lead-exposed workers from lead-acid battery manufacturing process. Int J Occup Environ Med. 2015;6(1):50-7. doi: 10.15171/ijoem.2015.436. [PubMed: 25588226].

15. Schwartz BS, Lee BK, Bandeen-Roche K, Stewart W, Bolla K, Links $\mathrm{J}$, et al. Occupational lead exposure and longitudinal decline in neurobehavioral test scores. Epidemiology. 2005;16(1):106-13. doi: 10.1097/01.ede.0000147109.62324.51. [PubMed: 15613953].

16. Shih RA, Glass TA, Bandeen-Roche K, Carlson MC, Bolla KI, Todd $A C$, et al. Environmental lead exposure and cognitive function in community-dwelling older adults. Neurology. 2006;67(9):1556-62. doi: 10.1212/01.wnl.0000239836.26142.c5. [PubMed: 16971698].

17. Barth A, Schaffer AW, Osterode W, Winker R, Konnaris C, Valic E, et al. Reduced cognitive abilities in lead-exposed men. Int Arch Occup Environ Health. 2002;75(6):394-8. doi: 10.1007/s00420-002-0329-1. [PubMed: 12070635].

18. Abdolmalki A, Farshad AA, Bagheri Yazdi SA, Nosrati M, Hamedani M, Mohamadi A. Study of lead effects on the mental health of tin laborers in a carmaking company of Tehran city 2007. Iran Occupational Health. 2009;6(2):26-30.

19. Fenga C, Gangemi S, Alibrandi A, Costa C, Micali E. Relationship between lead exposure and mild cognitive impairment. I Prev Med Hyg. 2016;57(4):E205-10. [PubMed: 28167858]. [PubMed Central: PMC5289032].

20. Winker R, Ponocny-Seliger E, Rudiger HW, Barth A. Lead exposure levels and duration of exposure absence predict neurobehavioral performance. Int Arch Occup Environ Health. 2006;79(2):123-7. doi: 10.1007/s00420-005-0031-1. [PubMed: 16088406].

21. Krieg EJ, Chrislip DW, Crespo CJ, Brightwell WS, Ehrenberg RL, Otto DA. The relationship between blood lead levels and neurobehavioral test performance in NHANES III and related occupational studies. Public Health Rep. 2005;120(3):240-51. doi: 10.1177/003335490512000305. [PubMed: 16134563]. [PubMed Central: PMC1497718].

22. Weisskopf MG, Wright RO, Schwartz J, Spiro A3, Sparrow D, Aro A, et al. Cumulative lead exposure and prospective change in cognition among elderly men: The VA Normative Aging Study. Am J Epidemiol. 2004;160(12):1184-93. doi: 10.1093/aje/kwh333. [PubMed: 15583371].

23. Shih RA, Hu H, Weisskopf MG, Schwartz BS. Cumulative lead dose and cognitive function in adults: A review of studies that measured both blood lead and bone lead. Environ Health Perspect. 2007;115(3):48392. doi: 10.1289/ehp.9786. [PubMed: 17431502]. [PubMed Central: PMC1849945]. 